\title{
EXPONENTIAL STABILISATION OF A TREE-SHAPED NETWORK OF STRINGS WITH VARIABLE COEFFICIENTS*
}

\author{
YAN NI GUO \\ Institute of Applied Mathematics, College of Science, Civil Aviation University of China, \\ Tianjin 300300, P.R. China \\ e-mail:gyann@126.com \\ and GEN QI XU \\ Department of Mathematics, Tianjin University, Tianjin 300072, P.R. China \\ e-mail:gqxu@tju.edu.cn
}

(Received 7 March 2010; revised 16 August 2010; accepted 30 November 2010;

first published online 10 March 2011)

\begin{abstract}
In this paper, we deal with a tree-shaped network of strings with a fixed root node. By imposing velocity feedback controllers on all vertices except the root node, we show that the spectrum of the system operator consists of all isolated eigenvalues of finite multiplicity and is distributed in a strip parallel to the imaginary axis under certain conditions. Moreover, we prove that there exists a sequence of eigenvectors and generalised eigenvectors that forms a Riesz basis with parentheses, and that the imaginary axis is not an asymptote of the spectrum. Thereby, we deduce that the system is exponentially stable.
\end{abstract}

2010 Mathematics Subject Classification. 93C20, 93D15, 35B35, 35P10, 47A70.

1. Introduction. In past decades, there had been extensive literature devoted to research on controllability and stabilisation of one-dimensional (1D) networks. For instance, we refer to earlier works $[\mathbf{5}, \mathbf{8}, \mathbf{9}, \mathbf{1 0}]$, recent works $[\mathbf{1}, \mathbf{2}, \mathbf{7}, \mathbf{1 8}, \mathbf{2 0}, \mathbf{2 2}, \mathbf{2 3}]$ and the references therein. The main tools used in the literature are the Hilbert uniqueness method, the multiplier approach and the Riesz basis approach. We observe that the models discussed in the papers mentioned above are mainly of uniform structure; there are few results on non-homogeneous structure of $1 \mathrm{D}$ networks. Maybe part of the reason for the non-uniform networks is that there is no solution formula such as D'Alembert formula for 1D wave equation, and the investigation of controllability and observability of the networks tends to be very difficult. Therefore, Dager and Zuazua in [7] proposed that, as an open problem, the control problem of the network of $1 \mathrm{D}$ strings with variable coefficients is an important subject in future study. In this paper, we will study the stabilisation problem of a tree-shaped network of strings with variable coefficients using the spectral method. Since controllability and stabilisation of the system depend not only on the physical characteristics that support the graphs but also on the structures of the graphs, we suppose that the tree-shaped network is comprised of three strings with one fixed vertex called the root node, and three vertices

*This research is supported by the Natural Science Foundation of China (60874034) and part by the Scientific Research Initiation Foundation of Civil Aviation University of China (08QD09X). 
that are imposed velocity feedback controllers. Although this model is simple, our approach can be applied to more complicated networks.

As a common problem in the investigation of networks by the Riesz basis method, to determine the spectral distribution of the closed-loop system is the first difficulty we encountered in the study, but it becomes much more difficult because of the variable coefficients. Even in single-string cases, the proof is also complicated (see [17]). Herein, we mainly employ the asymptotic analysis technique (see [11]) to overcome this difficulty. Using asymptotic analysis, we get the asymptotic distribution of the system operator's spectrum. The second difficulty comes from stability analysis of the system with variable coefficients, since the multiplier approach does not work for the networks. Here, we mainly use the Riesz basis approach as used in [19, 20, 22] to first obtain the spectrum-determined growth condition, and then to prove that the imaginary axis is not an asymptote of spectrum to obtain the stability. Although the proofs look like routine checks, the discussion of the Riesz basis property of eigenvectors and asymptote of spectrum have been difficult tasks in the spectral theory of linear operators.

Let $G=(V, E)$ be a simply connected graph, as defined by [4], where $V=$ $\left\{a, a_{1}, a_{2}, a_{3}\right\}$ is the vertex set and $E=\left\{e_{1}, e_{2}, e_{3}\right\}$ is the edge set. a is the common vertex, named interior node of $G$, and the vertices $a_{1}, a_{2}$ and $a_{3}$, each receiving only one edge, are called boundary nodes of $G$. Assume that one of the boundary nodes, say $a_{1}$, is fixed and the others are free. Suppose that edge $e_{i}(i=1,2,3)$ has a finite arc length $\ell_{i}$, which can be parameterised by its arc length, i.e. a mapping $\pi_{i}$ defined by $\pi_{i}:\left[0, \ell_{i}\right] \longrightarrow e_{i}, i=1,2,3$. So, edge $e_{i}$ can identify an interval $\left[0, \ell_{i}\right](i=1,2,3)$.

Let the strings expand on $G$, whose equilibrium positions coincide with $G$. Denote the displacement of $i_{t h}$ string in position $\pi_{i}(x) \in e_{i}$ at time $t, i=1,2,3$ with $u_{i}(x, t)$. The motion of the string on edge $e_{i}$ is governed by

$$
\rho_{i}(x) \partial_{t}^{2} u_{i}(x, t)-\partial_{x}\left[\sigma_{i}(x) \partial_{x} u_{i}(x, t)\right]+q_{i}(x) u_{i}(x, t)=0, x \in\left(0, \ell_{i}\right), t>0,
$$

where $\rho_{i}(x)$ and $\sigma_{i}(x)>0$ are the mass density and the modulus of elasticity of the $i_{t h}$ string, respectively, and $q_{i}(x)$ is the rigidity coefficient. Suppose that the strings system satisfies the following conditions:

(1) At the common node $a$, impose geometric continuity conditions: $u_{1}(0, t)=$ $u_{2}(0, t)=u_{3}(0, t)$, and the dynamic condition (Kirchhoff law): $\sum_{i=1}^{3} \sigma_{i}(0) \partial_{x} u_{i}(0, t)=$ $h \partial_{t} u_{1}(0, t), h>0$.

(2) On the boundary vertices, impose the Dirichlet condition and the damping conditions: $u_{1}\left(\ell_{1}\right)=0$ and $\sigma_{i}\left(\ell_{i}\right) \partial_{x} u_{i}\left(\ell_{i}, t\right)=-k_{i} \partial_{t} u_{i}\left(\ell_{i}, t\right), k_{i}>0, i=2,3$.

In addition, we assume that the initial conditions of the system are $u_{i}(x, 0)=u_{i 0}(x)$ and $\partial_{t} u_{i}(x, 0)=u_{i 1}(x), i=1,2,3$. Thus, the dynamic behaviour of the network of strings is described by the partial differential equations

$$
\left\{\begin{array}{l}
\rho_{i}(x) \partial_{t t}^{2} u_{i}(x, t)-\partial_{x}\left[\sigma_{i}(x) \partial_{x} u_{i}(x, t)\right]+q_{i}(x) u_{i}(x, t)=0, x \in\left(0, \ell_{i}\right), t>0 ; \\
u_{1}(0, t)=u_{2}(0, t)=u_{3}(0, t) \\
u_{1}\left(\ell_{1}, t\right)=0 \\
\sum_{i=1}^{3} \sigma_{i}(0) \partial_{x} u_{i}(0, t)=h \partial_{t} u_{1}(0, t), h>0 \\
\sigma_{i}\left(\ell_{i}\right) \partial_{x} u_{i}\left(\ell_{i}, t\right)=-k_{i} \partial_{t} u_{i}\left(\ell_{i}, t\right), i=2,3 ; \\
u_{i}(x, 0)=u_{i 0}(x), \quad \partial_{t} u_{i}(x, 0)=u_{i 1}(x), i=1,2,3 .
\end{array}\right.
$$


The remainder of the paper is organised as follows. In Section 2, we discuss the well-posedness and the asymptotic stability of the system (1.1). In Section 3, we focus on the spectral distribution of the system operator $\mathcal{A}$. By a detailed asymptotic analysis, we prove that the spectrum of $\mathcal{A}$ is distributed in a strip parallel to the imaginary axis. Finally, in Section 4, we prove that the generalised eigenvectors of $\mathcal{A}$ are complete in the state space $\mathcal{H}$, and there is a sequence of generalised eigenvectors that form a Riesz basis with parentheses. In particular, we prove that the imaginary axis is not an asymptote of the spectrum, and hence, we get the exponential stability of the system.

2. Well-posedness of the system. In order to study the well-posedness of the system (1.1), we first formulate (1.1) into an evolution equation in a Hilbert state space. Let $H^{k}\left(0, \ell_{i}\right)(i=1,2,3, k=1,2)$ be the usual Sobolev space and $L^{2}\left(0, \ell_{i}\right)(i=1,2,3)$ be the usual Hilbert space.

Set $\quad X:=\left\{u=\left(u_{i}\right)_{i=1}^{3} \in \Pi_{i=1}^{3} H^{1}\left(0, \ell_{i}\right) \mid u_{i}(0)=u_{j}(0), \forall i, j=1,2,3 ; u_{1}\left(\ell_{1}\right)=0\right\}$ endowed with the inner product

$$
\langle u, v\rangle_{X}=\sum_{i=1}^{3} \int_{0}^{\ell_{i}}\left(\sigma_{i}(x) u_{i}^{\prime}(x) \overline{v_{i}^{\prime}(x)}+q_{i}(x) u_{i}(x) \overline{v_{i}(x)}\right) d x, \quad \forall u, v \in X
$$

here $u^{\prime}=\frac{d u}{d x}$. Assume that $\rho_{i}(x), \sigma_{i}(x), q_{i}(x) \in H^{2}\left(0, \ell_{i}\right), i=1,2,3$ are positive functions.

Let state space $\mathcal{H}=X \times \Pi_{i=1}^{3} L^{2}\left(0, \ell_{i}\right)$ equipped with the norm

$$
\|(u, v)\|_{\mathcal{H}}^{2}=\sum_{i=1}^{3} \int_{0}^{\ell_{i}}\left[\sigma_{i}(x)\left|u_{i}^{\prime}(x)\right|^{2}+q_{i}(x)\left|u_{i}(x)\right|^{2}+\rho_{i}(x)\left|v_{i}(x)\right|^{2}\right] d x .
$$

Obviously, $(\mathcal{H},\|\cdot\|)$ is a Hilbert space.

Define the operator $\mathcal{A}$ in $\mathcal{H}$ by

$$
\mathcal{A}\left(\begin{array}{l}
u \\
v
\end{array}\right)=\left(\begin{array}{c}
v \\
\left\{\rho_{i}^{-1}(x)\left[\left[\sigma_{i}(x) u_{i}^{\prime}(x)\right]^{\prime}-q_{i}(x) u_{i}(x)\right]\right\}_{i=1}^{3}
\end{array}\right)
$$

with domain

$$
D(\mathcal{A})=\left\{\begin{array}{l}
(u, v) \in X \cap \Pi_{i=1}^{3} H^{2}\left(0, \ell_{i}\right) \times X \\
\left.\sum_{i=1}^{3} \sigma_{i}(0) u_{i}^{\prime}(0)=h v_{1}(0), \sigma_{i}\left(\ell_{i}\right) u_{i}^{\prime} \ell_{i}\right)=-k_{i} v_{i}\left(\ell_{i}\right), i=2,3
\end{array}\right\}
$$

Then, we can rewrite (1.1) into an evolutionary equation in $\mathcal{H}$

$$
\left\{\begin{array}{l}
\frac{d Y(t)}{d t}=\mathcal{A} Y(t), \quad t>0 \\
Y(0)=Y_{0}
\end{array}\right.
$$

where $Y(t)=\left(u(\cdot, t), \partial_{t} u(\cdot, t)\right)^{T}, \quad Y(0)=\left(U_{0}, U_{1}\right)^{T} \in \mathcal{H}, U_{0}=\left\{u_{i 0}\right\}_{i=1}^{3}, U_{1}=\left\{u_{i 1}\right\}_{i=1}^{3}$.

TheOrem 2.1. Let $\mathcal{A}$ be defined by (2.1)-(2.2). Then, $\mathcal{A}$ is a closed and densely defined linear operator in $\mathcal{H}$, in addition, $0 \in \rho(\mathcal{A})$ and $\mathcal{A}^{-1}$ is compact. 
Proof. It is easy to check that $\mathcal{A}$ is a densely defined and closed linear operator. Here, we only need to prove that $0 \in \rho(\mathcal{A})$ and $\mathcal{A}^{-1}$ is compact.

Firstly, we show that $\mathcal{A}^{-1}$ exists. Let $(u, v) \in D(\mathcal{A})$ such that $\mathcal{A}(u, v)=0$, then we have $v=0$ and $u_{i}(x)$ satisfies

$$
\begin{aligned}
& {\left[\sigma_{i}(x) u_{i}^{\prime}(x)\right]^{\prime}-q_{i}(x) u_{i}(x)=0, x \in\left(0, \ell_{i}\right), i=1,2,3} \\
& u_{1}\left(\ell_{1}\right)=0 \\
& u_{i}\left(\ell_{i}\right)=0, i=2,3 \\
& \sum_{i=1}^{3} \sigma_{i}(0) u_{i}^{\prime}(0)=0 \\
& \sigma_{i}\left(\ell_{i}\right) u_{i}^{\prime}\left(\ell_{i}\right)=0, i=2,3 .
\end{aligned}
$$

By multiplying equation (2.4) by $\overline{u_{i}}$, integrating over the interval $\left[0, \ell_{i}\right]$ and adding them up, and using the boundary and node conditions (2.5)-(2.8), we deduce

$$
0=-\sum_{i=1}^{3} \int_{0}^{\ell_{i}}\left[\sigma_{i}(x)\left|u_{i}^{\prime}(x)\right|^{2}+q_{i}(x)\left|u_{i}(x)\right|^{2}\right] d x .
$$

Since $\sigma_{i}(x)>0, q_{i}(x)>0$, we get from the above equality that $u_{i}(x) \equiv 0, i=1,2,3$. Therefore, $(u, v) \equiv 0$, which implies that $\mathcal{A}$ is injective.

We claim that $\mathcal{A}$ is surjective. Indeed, for any $(f, g) \in \mathcal{H}$, we consider solvability of equation $\mathcal{A}(u, v)=(f, g)$, i.e.,

$$
\left\{\begin{array}{l}
v_{i}(x)=f_{i}(x) \\
{\left[\sigma_{i}(x) u_{i}^{\prime}(x)\right]^{\prime}-q_{i}(x) u_{i}(x)=g_{i}(x) \rho_{i}(x), i=1,2,3} \\
u_{1}\left(\ell_{1}\right)=0 ; \quad u_{i}(0)=u_{j}(0), i=1,2,3 \\
\sum_{i=1}^{3} \sigma_{i}(0) u_{i}^{\prime}(0)=h v_{1}(0) \\
\sigma_{i}\left(\ell_{i}\right) u_{i}^{\prime}\left(\ell_{i}\right)=-k_{i} v_{i}\left(\ell_{i}\right), i=2,3
\end{array}\right.
$$

By multiplying the second equality by $\overline{\phi_{i}(x)}$, where $\phi_{i}(x)(i=1,2,3)$ are the test function, integrating over $\left(0, \ell_{i}\right)$ and integration by parts, we get

$$
\begin{aligned}
\sum_{i=1}^{3} \int_{0}^{\ell_{i}} g_{i}(x) \rho_{i}(x) \overline{\phi_{i}(x)} d x= & -\sum_{i=1}^{3} \int_{0}^{\ell_{i}}\left[\sigma_{i}(x) u_{i}^{\prime}(x) \overline{\phi_{i}^{\prime}(x)}+q_{i}(x) u_{i}(x) \overline{\phi_{i}(x)}\right] d x \\
& +\left.\sum_{i=1}^{3} \sigma_{i}(x) u_{i}^{\prime}(x) \overline{\phi_{i}(x)}\right|_{0} ^{\ell_{i}} .
\end{aligned}
$$

Since $(u, v) \in D(\mathcal{A})$, we have

$$
\left.\sum_{i=1}^{3} \sigma_{i}(x) u_{i}^{\prime}(x) \overline{\phi_{i}(x)}\right|_{0} ^{\ell_{i}}=-\sum_{i=2}^{3} k_{i} f_{i}\left(\ell_{i}\right) \overline{\phi_{i}\left(\ell_{i}\right)}-h f_{1}(0) \overline{\phi(0)} .
$$


For any $w, z \in X$, we define the bilinear form $B(w, z)$ by

$$
B(w, z)=\sum_{i=1}^{3} \int_{0}^{\ell_{i}}\left[\sigma_{i}(x) w_{i}^{\prime}(x) \overline{z_{i}^{\prime}(x)}+q_{i}(x) w_{i}(x) \overline{z_{i}(x)}\right] d x .
$$

Clearly, $|B(w, z)| \leq\|w\|_{X} \cdot\|z\|_{X}, \forall w, z \in X$. In addition, $B$ is coercive, since $B(w, w)=$ $\|w\|_{X}^{2}$. The Lax-Milgram's theorem asserts that there exist $u=\left\{u_{i}(x)\right\}_{i=1}^{3}$ such that the equations

$$
\left[\sigma_{i}(x) u_{i}^{\prime}(x)\right]^{\prime}-q_{i}(x) u_{i}(x)=g_{i}(x) \rho_{i}(x), \quad i=1,2,3
$$

are fulfilled. This implies that $u \in \Pi_{i=1}^{3} H^{2}\left(0, \ell_{i}\right)$. Hence, $u \in \Pi_{i=1}^{3} H^{2}\left(0, \ell_{i}\right) \cap X$. Since $v=f$, we have found $(u, v)=(u, f) \in D(\mathcal{A})$ such that $\mathcal{A}(u, v)=(f, g)$ for given $(f, g) \in \mathcal{H}$.

Summarising the above discussions, the closed operator theorem ensures that $\mathcal{A}^{-1}$ is bounded. Consequently, $0 \in \rho(\mathcal{A})$. Note that $D(\mathcal{A}) \subset \Pi_{i=1}^{3} H^{2}\left(0, \ell_{i}\right) \times X$, so $\mathcal{A}^{-1}$ is compact owing to compact embedding theorem.

As a result of compact operator, we have the following corollary.

COROLlary 2.1. The spectrum of $\mathcal{A}$ consists of all isolated eigenvalues of finite multiplicity. (see [6].)

Corollary 2.2. Let $\mathcal{A}$ be defined by (2.1)-(2.2), then $\mathcal{A}$ generates a $C_{0}$-semigroup of contractions on $\mathcal{H}$ and hence the system (2.3) is well-posed in $\mathcal{H}$.

Proof. For any $(u, v) \in D(\mathcal{A})$, a direct calculation shows that

$$
\Re\langle\mathcal{A}(u, v),(u, v)\rangle_{\mathcal{H}}=-\left[\sum_{i=2}^{3} k_{i}\left|v_{i}\left(\ell_{i}\right)\right|^{2}+h\left|v_{1}(0)\right|^{2}\right] \leq 0,
$$

which implies that $\mathcal{A}$ is dissipative.

Theorem 2.1 together with dissipation of $\mathcal{A}$ assert that $\mathcal{A}$ generates a $C_{0}$-semigroup of contractions on $\mathcal{H}$ thanks to the Lumer-Phillips theorem (see [12]).

3. Asymptotic distribution of spectrum of $\mathcal{A}$. In this section, we will discuss the asymptotic distribution of the spectrum of $\mathcal{A}$. Due to Corollary 2.1, we need only to discuss the eigenvalue problem of $\mathcal{A}$.

Let $\lambda \in \mathbb{C}$ and let there be a non-zero vector $(u, v) \in D(\mathcal{A})$ such that $(\lambda I-$ $\mathcal{A})(u, v)=0$. From this, we can deduce that $v=\lambda u$ and $u=\left(u_{i}\right)_{i=1}^{3}$ satisfy the following equations

$$
\begin{aligned}
& \lambda^{2} \rho_{i}(x) u_{i}(x)-\left[\left(\sigma_{i}(x) u_{i}^{\prime}(x)\right)^{\prime}-q_{i}(x) u_{i}(x)\right]=0, x \in\left(0, \ell_{i}\right), i=1,2,3 \\
& u_{1}\left(\ell_{1}\right)=0 \\
& \sigma_{i}\left(\ell_{i}\right) u_{i}^{\prime}\left(\ell_{i}\right)=-k_{i} \lambda u_{i}\left(\ell_{i}\right), i=2,3 \\
& u_{1}(0)=u_{2}(0)=u_{3}(0) \\
& \sum_{i=1}^{3} \sigma_{i}(0) u_{i}^{\prime}(0)=\lambda h u_{1}(0)
\end{aligned}
$$


In what follows, we will transform equation (3.1) into a standard form so that we can get an asymptotic expression of the solution in $\lambda$. The process will be carried out in three steps.

Step 1. By a variable change, transform equation (3.1) into the form without the first-order derivative of $u_{i}(x)(i=1,2,3)$.

Define new functions $y_{i}(x)$ by $y_{i}(x):=\sqrt{\sigma_{i}(x)} u_{i}(x)(i=1,2,3)$. Substituting them into (3.1) yields

$$
\frac{d^{2} y_{i}}{d x^{2}}(x)-\lambda^{2} \frac{\rho_{i}(x)}{\sigma_{i}(x)} y_{i}(x)-b_{i}(x) \frac{\rho_{i}(x)}{\sigma_{i}(x)} y_{i}(x)=0, \quad i=1,2,3
$$

where

$$
b_{i}(x)=-\left[\frac{1}{4}\left(\frac{\frac{d \sigma_{i}}{d x}(x)}{\sigma_{i}(x)}\right)^{2}-\frac{1}{2}\left(\frac{\frac{d^{2} \sigma_{i}}{d x^{2}}}{\sigma_{i}}\right)(x)-\frac{q_{i}(x)}{\sigma_{i}(x)}\right] \frac{\sigma_{i}(x)}{\rho_{i}(x)} .
$$

(3.6) is exactly of the form without the first derivative.

Corresponding to the new function $y_{i}(x),(3.2)-(3.5)$ become

$$
\begin{aligned}
& y_{1}\left(\ell_{1}\right)=0 \\
& \sigma_{i}\left(\ell_{i}\right) \frac{d y_{i}}{d x}\left(\ell_{i}\right)+\left[k_{i} \lambda-\frac{1}{2} \sigma_{i x}\left(\ell_{i}\right)\right] y_{i}\left(\ell_{i}\right)=0, i=2,3 \\
& \left(\sigma_{1}(0)\right)^{-1 / 2} y_{1}(0)=\left(\sigma_{2}(0)\right)^{-1 / 2} y_{2}(0)=\left(\sigma_{3}(0)\right)^{-1 / 2} y_{3}(0) \\
& \sum_{i=1}^{3}\left(\sigma_{i}(0)\right)^{-1 / 2} \sigma_{i}(0) \frac{d y_{i}}{d x}(0)-\frac{1}{2} \sum_{i=1}^{3}\left(\sigma_{i}(0)\right)^{-1 / 2} \sigma_{i x}(0) y_{i}(0)=\lambda h\left(\sigma_{1}(0)\right)^{-1 / 2} y_{1}(0) .
\end{aligned}
$$

Step 2. By changes of variable, transform equation (3.6) into a standard form so that the coefficient before the unknown function is $-\lambda^{2}$.

Introduce a new variable

$$
\xi_{i}(x)=\int_{0}^{x} \sqrt{\frac{\rho_{i}(t)}{\sigma_{i}(t)}} d t, \quad x \in\left(0, \ell_{i}\right)
$$

and define new functions $w_{i}\left(\xi_{i}\right), i=1,2,3$ by

$$
w_{i}\left(\xi_{i}\right):=\frac{1}{\sqrt{x^{\prime}}} y_{i}\left(x\left(\xi_{i}\right)\right)
$$

where $x\left(\xi_{i}\right)$ is the inverse function of $\xi_{i}(x)$. Here, the prime always denote $f^{\prime}(\xi)=\frac{d f}{d \xi}$.

Putting

$$
\begin{aligned}
& m_{i}:=\int_{0}^{\ell_{i}} \sqrt{\frac{\rho_{i}(t)}{\sigma_{i}(t)}} d t \\
& \phi_{i}\left(\xi_{i}\right):=-\frac{3}{4}\left(x^{\prime}\left(\xi_{i}\right)\right)^{-2}\left(x^{\prime \prime}\left(\xi_{i}\right)\right)^{2}+\frac{1}{2}\left(x^{\prime}\left(\xi_{i}\right)\right)^{-1} x^{\prime \prime \prime}\left(\xi_{i}\right),
\end{aligned}
$$


the equation (3.6) and boundary and connection conditions (3.8)-(3.11) are changed into

$$
\begin{aligned}
& w_{i}^{\prime \prime}\left(\xi_{i}\right)-\lambda^{2} w_{i}\left(\xi_{i}\right)=\left[b_{i}\left(x\left(\xi_{i}\right)\right)-\phi_{i}\left(\xi_{i}\right)\right] w_{i}\left(\xi_{i}\right), \xi_{i} \in\left(0, m_{i}\right), i=1,2,3 \\
& w_{1}\left(m_{1}\right)=0 \\
& \sigma_{i}\left(\ell_{i}\right) w_{i}^{\prime}\left(m_{i}\right)+\left[c_{i}\left(\ell_{i}\right)+k_{i} \lambda\left(\frac{\rho_{i}\left(\ell_{i}\right)}{\sigma_{i}\left(\ell_{i}\right)}\right)^{-1 / 2}\right] w_{i}\left(m_{i}\right)=0, i=2,3 \\
& \left(\rho_{1}(0) \sigma_{1}(0)\right)^{-1 / 4} w_{1}(0)=\left(\rho_{2}(0) \sigma_{2}(0)\right)^{-1 / 4} w_{2}(0)=\left(\rho_{3}(0) \sigma_{3}(0)\right)^{-1 / 4} w_{3}(0) \\
& \sum_{i=1}^{3}\left(\rho_{i}(0) \sigma_{i}(0)\right)^{1 / 4} w_{i}^{\prime}(0)+\sum_{i=1}^{3} d_{i}(0) w_{i}(0)=\lambda h\left(\rho_{1}(0) \sigma_{1}(0)\right)^{-1 / 4} w_{1}(0)
\end{aligned}
$$

where $b_{i}(x)$ and $\phi_{i}\left(\xi_{i}\right)$ are given by (3.7) and (3.13), respectively, and

$$
\begin{aligned}
& c_{i}(x):=-\frac{1}{4}\left(\frac{\rho_{i}(x)}{\sigma_{i}(x)}\right)^{-3 / 2} \sigma_{i}(x)\left(\frac{\rho_{i}}{\sigma_{i}}\right)_{x}(x)-\frac{1}{2} \sigma_{i x}(x)\left(\frac{\rho_{i}(x)}{\sigma_{i}(x)}\right)^{-1 / 2}, i=2,3 ; \\
& d_{i}(x):=-\frac{1}{4} \sigma_{i}(x)^{1 / 2}\left(\frac{\rho_{i}(x)}{\sigma_{i}(x)}\right)^{-5 / 4}\left(\frac{\rho_{i}}{\sigma_{i}}\right)_{x}(x)-\frac{1}{2} \sigma_{i x}(x)\left(\rho_{i}(x) \sigma_{i}(x)\right)^{-1 / 4}, i=1,2,3 .
\end{aligned}
$$

Thus, (3.14) is the desired standard form.

Observing that the transforms introduced above are reversible, the following result is obvious.

Proposition 3.1. The eigenvalue problem of (3.1)-(3.5) is equivalent to that of (3.14)-(3.18).

Step 3. Get the asymptotic expression of the solution in $\lambda$.

According to the theory of ordinary differential equations, there exist two linearly independent solutions $F_{i}\left(\lambda, \xi_{i}\right)$ and $\Psi_{i}\left(\lambda, \xi_{i}\right)$ to (3.14). For $\lambda \in \mathbb{C}$ with $|\lambda| \geq \delta>0$, they have the asymptotic expressions in $\lambda$ (see, [11, Theorem 1, p. 49]):

$$
\begin{aligned}
F_{i}\left(\lambda, \xi_{i}\right)=e^{\lambda \xi_{i}}\left[1+O\left(\lambda^{-1}\right)\right], & \Psi_{i}\left(\lambda, \xi_{i}\right) & =e^{-\lambda \xi_{i}}\left[1+O\left(\lambda^{-1}\right)\right] \\
F_{i}^{\prime}\left(\lambda, \xi_{i}\right)=\lambda e^{\lambda \xi_{i}}\left[1+O\left(\lambda^{-1}\right)\right], & \Psi_{i}^{\prime}\left(\lambda, \xi_{i}\right) & =\lambda e^{-\lambda \xi_{i}}\left[-1+O\left(\lambda^{-1}\right)\right] .
\end{aligned}
$$

Thus, the general solution of (3.14) is given by

$$
\begin{aligned}
w_{i}\left(\lambda, \xi_{i}\right) & =A_{i}(\lambda) F_{i}\left(\lambda, \xi_{i}\right)+B_{i}(\lambda) \Psi_{i}\left(\lambda, \xi_{i}\right) \\
& =A_{i}(\lambda) e^{\lambda \xi_{i}}\left[1+O\left(\lambda^{-1}\right)\right]+B_{i}(\lambda) e^{-\lambda \xi_{i}}\left[1+O\left(\lambda^{-1}\right)\right]
\end{aligned}
$$

where $A_{i}(\lambda)$ and $B_{i}(\lambda)$ are coefficients dependent on $\lambda$. 
Now, we are in a position to determine the asymptotic distribution of the spectrum of $\mathcal{A}$. Inserting (3.23) into (3.15)-(3.18) lead to

$$
\begin{aligned}
& A_{1}(\lambda)[1]_{1} e^{\lambda m_{1}}+B_{1}(\lambda)[1]_{1} e^{-\lambda m_{1}}=0 \\
& \left.A_{i}(\lambda) K_{i}^{+}\right]_{1} e^{\lambda m_{i}}+B_{i}(\lambda)\left[K_{i}^{-}\right]_{1} e^{-\lambda m_{i}}=0, i=2,3 \\
& {\left[\widehat{\rho}_{1}\right]_{1}\left[A_{1}(\lambda)+B_{1}(\lambda)\right]=\left[\widehat{\rho}_{2}\right]_{1}\left[A_{2}(\lambda)+B_{2}(\lambda)\right]=\left[\widehat{\rho}_{3}\right]_{1}\left[A_{3}(\lambda)+B_{3}(\lambda)\right]} \\
& A_{1}(\lambda)\left[H_{1}^{-}\right]_{1}+\sum_{i=2}^{3} A_{i}(\lambda)\left[H_{i}^{-}\right]_{1}-B_{1}(\lambda)\left[H_{1}^{+}\right]_{1}-\sum_{i=2}^{3} B_{i}(\lambda)\left[H_{i}^{+}\right]_{1}=0
\end{aligned}
$$

where the notation $[a]_{1}=a+O\left(\lambda^{-1}\right)$ and

$$
\begin{aligned}
& K_{i}^{+}=k_{i}\left(\frac{\rho_{i}\left(\ell_{i}\right)}{\sigma_{i}\left(\ell_{i}\right)}\right)^{-1 / 2}+\sigma_{i}\left(\ell_{i}\right), K_{i}^{-}=k_{i}\left(\frac{\rho_{i}\left(\ell_{i}\right)}{\sigma_{i}\left(\ell_{i}\right)}\right)^{-1 / 2}-\sigma_{i}\left(\ell_{i}\right), \quad i=2,3, \\
& H_{1}^{-}=\frac{\sqrt{\rho_{i}(0) \sigma_{i}(0)}-h}{\sqrt[4]{\rho_{i}(0) \sigma_{i}(0)}}, \quad H_{1}^{+}=\frac{\sqrt{\rho_{i}(0) \sigma_{i}(0)}+h}{\sqrt[4]{\rho_{i}(0) \sigma_{i}(0)}}, \\
& H_{i}^{ \pm}=\left(\rho_{i}(0) \sigma_{i}(0)\right)^{1 / 4}, i=2,3, \quad \widehat{\rho}_{i}=\left(\rho_{i}(0) \sigma_{i}(0)\right)^{-1 / 4}, i=1,2,3,
\end{aligned}
$$

and denoted by $\Delta(\lambda)$ the coefficients matrix of algebraic equations (3.24)-(3.27), i.e.,

$$
\Delta(\lambda)=\left(\begin{array}{cccccc}
e^{\lambda m_{1}}[1]_{1} & 0 & 0 & e^{-\lambda m_{1}}[1]_{1} & 0 & 0 \\
0 & {\left[K_{2}^{+}\right]_{1} e^{\lambda m_{2}}} & 0 & 0 & {\left[K_{2}^{-}\right]_{1} e^{-\lambda m_{2}}} & 0 \\
0 & 0 & {\left[K_{3}^{+}\right]_{1} e^{\lambda m_{3}}} & 0 & 0 & {\left[K_{3}^{-}\right]_{1} e^{-\lambda m_{3}}} \\
{\left[\widehat{\rho}_{1}\right]_{1}} & -\left[\widehat{\rho}_{2}\right]_{1} & 0 & {\left[\widehat{\rho}_{1}\right]_{1}} & -\left[\widehat{\rho}_{2}\right]_{1} & 0 \\
{\left[\widehat{\rho}_{1}\right]_{1}} & 0 & -\left[\widehat{\rho}_{3}\right]_{1} & {\left[\widehat{\rho}_{1}\right]_{1}} & 0 & -\left[\widehat{\rho}_{3}\right]_{1} \\
{\left[H_{1}^{-}\right]_{1}} & {\left[H_{2}^{-}\right]_{1}} & {\left[H_{3}^{-}\right]_{1}} & -\left[H_{1}^{+}\right]_{1} & -\left[H_{2}^{+}\right]_{1} & -\left[H_{3}^{+}\right]_{1}
\end{array}\right)
$$

Obviously, the eigenvalue problem has a non-zero solution if and only if det $\Delta(\lambda)=0$.

A direct calculation gives

$$
\lim _{\Re \lambda \rightarrow-\infty} \frac{\operatorname{det} \Delta(\lambda)}{e^{-\lambda \sum_{i=1}^{3} m_{i}}}=-\prod_{i=2}^{3} K_{i}^{-}\left\{\sum_{i=1}^{3} \prod_{j=1, j \neq i}^{3} \widehat{\rho}_{j} H_{i}^{-}\right\} \neq 0
$$

provided that

$$
\left\{\begin{array}{l}
h \in \mathbb{R} \text { such that } \sum_{i=1}^{3} \prod_{j=1, j \neq i}^{3} \widehat{\rho}_{j} H_{i}^{-} \neq 0 \\
k_{i} \neq \sqrt{\rho_{i}\left(\ell_{i}\right) \sigma_{i}\left(\ell_{i}\right)}, i=2,3
\end{array}\right.
$$

Similarly, we have

$$
\lim _{\Re \lambda \rightarrow+\infty} \frac{\operatorname{det} \Delta(\lambda)}{e^{\lambda \sum_{i=1}^{3} m_{i}}}=-\prod_{i=2}^{3} K_{i}^{+}\left\{\sum_{i=1}^{3} \prod_{j=1, j \neq i}^{3} \widehat{\rho}_{j} H_{i}^{+}\right\} \neq 0 .
$$

Summarising the above discussions, we achieve the following result. 
Theorem 3.1. Let $\mathcal{A}$ be defined by (2.1)-(2.2). Then, $\lambda \in \mathbb{C}$ is an eigenvalue of $\mathcal{A}$ if and only if det $\Delta(\lambda)=0$. If $k_{i}, i=2,3$ and h satisfy condition (3.31), then the spectrum of $\mathcal{A}$ is distributed in a strip parallel to the imaginary axis. Moreover, $\sigma(\mathcal{A})$ is a union of finitely separable sets.

Proof. Obviously, $\lambda$ is an eigenvalue of $\mathcal{A}$, so $\operatorname{det} \Delta(\lambda)=0$. Conversely, if $\lambda \in \mathbb{C}$ such that det $\Delta(\lambda)=0$, the algebraic equations (3.24)-(3.27) have non-zero solution $A_{i}(\lambda), B_{i}(\lambda), i=1,2,3$. By Proposition 3.1, the eigenvalue problem has a non-zero solution, and hence $\lambda$ is an eigenvalue of $\mathcal{A}$.

Observe that if the condition (3.31) is fulfilled, then (3.30) and (3.32) show that there exist positive constants $A, B$ and $p$ such that

$$
A \leq \frac{|\operatorname{det} \Delta(\lambda)|}{e^{|\Re \lambda|} \sum_{i=1}^{3} m_{i}} \leq B, \quad|\Re \lambda|>p .
$$

This implies that the spectrum of $\mathcal{A}$ is distributed in a strip parallel to the imaginary axis. Based on the above inequality, Levin's theorem [3] asserts that $\sigma(\mathcal{A})$ is a union of finitely many separable sets. The proof is then complete.

4. The exponential stability of the system. In this section, we will prove the exponential stability of the system (2.3). To this end, we show that there is a sequence of eigenvector and generalised eigenvectors of $\mathcal{A}$ that forms a Riesz basis with parentheses for $\mathcal{H}$, which implies the spectrum-determined growth condition. To show the Riesz basis property of (2.3), the key point is to deal with the completeness of the eigenvectors and generalised eigenvectors of $\mathcal{A}$ in $\mathcal{H}$.

There have been various methods to prove the completeness of eigenvectors and generalised eigenvectors of linear operators (see [11] and [13]). For the earlier works on the completeness for non-self-adjoint operators as well as differential equations with parameters in the boundary conditions, we refer to [14] and [15], in which the authors gave the basic idea and methods.

The following proposition is a special case in [14] and [15] of the generator of strongly continuous semigroups, which is stated in [19].

Proposition 4.1. Let $\mathcal{A}$ be the generator of a $C_{0}$-semigroup in a Hilbert space $\mathcal{H}$. Assume that $\mathcal{A}$ is discrete and, for $\lambda \in \rho\left(\mathcal{A}^{*}\right), R\left(\lambda, \mathcal{A}^{*}\right)$ is of the form

$$
R\left(\lambda, \mathcal{A}^{*}\right) x=\frac{G(\lambda) x}{F(\lambda)}, \quad \forall x \in \mathcal{H},
$$

where for each $x \in \mathcal{H}, G(\lambda) x$ is an $\mathcal{H}$-valued entire function with the order less than or equal to $\rho_{1}$, and $F(\lambda)$ is a scalar entire function of order $\rho_{2}$. Let $\rho=\max \left\{\rho_{1}, \rho_{2}\right\}<\infty$ and $n \in \mathbb{N}$ such that $n-1 \leq \rho<n$. If there are $n+1$ rays $\gamma_{j}, j=0,1, \cdots, n$ on the complex plane

$$
\arg \gamma_{0}=\frac{\pi}{2}<\arg \gamma_{1} \leq \arg \gamma_{2} \leq \cdots \leq \arg \gamma_{n}=\frac{3 \pi}{2}
$$


with

$$
\arg \gamma_{j+1}-\arg \gamma_{j} \leq \frac{\pi}{n}, \quad 0 \leq j \leq n-1
$$

such that $R\left(\lambda, \mathcal{A}^{*}\right) x$ is bounded on each ray $\gamma_{j}, 0 \leq j \leq n$ as $|\lambda| \rightarrow \infty$, for any $x \in \mathcal{H}$. Then $\operatorname{Sp}(\mathcal{A})=\operatorname{Sp}\left(\mathcal{A}^{*}\right)=\mathcal{H}$, where $\operatorname{Sp}(\mathcal{A})$ is the closed subspace spanned by all generalised eigenvectors of $\mathcal{A}$.

THEOREM 4.1. Let $\mathcal{A}$ be defined as (2.1)-(2.2). Then, the system of eigenvectors and generalised eigenvectors of $\mathcal{A}$ is complete in $\mathcal{H}$.

Proof. We complete the proof with three steps.

Step 1. Given arbitrary $(f, g) \in \mathcal{H},\left\|R\left(\lambda, \mathcal{A}^{*}\right)(f, g)\right\|$ is bounded as $\lambda \rightarrow-\infty$.

To prove this assertion, we introduce an auxiliary operator $\mathcal{A}_{0}$ defined by $\mathcal{A}_{0}(f, g):=\mathcal{A}(f, g)$ for any $(f, g) \in D\left(\mathcal{A}_{0}\right)$, where the domain

$$
D\left(\mathcal{A}_{0}\right):=\left\{\begin{array}{l|l}
(u, v) \in \mathcal{H} & \begin{array}{l}
v \in X, u_{i} \in H^{2}\left(0, \ell_{i}\right), \sum_{i=1}^{3} \sigma_{i}(0) u_{i}^{\prime}(0)=0, \\
\sigma_{i}\left(\ell_{i}\right) u_{i}^{\prime}\left(\ell_{i}\right)=0, i=2,3
\end{array}
\end{array}\right\} .
$$

$\mathcal{A}_{0}$ is a skew-adjoint operator in $\mathcal{H}$, and hence $\left\|R\left(\lambda, \mathcal{A}_{0}^{*}\right)\right\| \leq \frac{1}{|\lambda|}, \quad \forall \lambda \in \mathbb{R} \backslash\{0\}$.

Let $\lambda \in \rho\left(\mathcal{A}^{*}\right) \cap \mathbb{R}^{-}$and $(f, g) \in \mathcal{H}$. We write

$$
(u, v)=: R\left(\lambda, \mathcal{A}_{0}^{*}\right)(f, g), \quad(w, z)=: R\left(\lambda, \mathcal{A}^{*}\right)(f, g)-(u, v),
$$

where $\mathcal{A}^{*}$ is the adjoint operator of $\mathcal{A}$.

Thus

$$
\left\|R\left(\lambda, \mathcal{A}^{*}\right)(f, g)\right\|=\|(w, z)+(u, v)\| \leq\|(w, z)\|+\frac{1}{|\lambda|}\|(f, g)\| .
$$

A direct calculation gives

$$
\begin{aligned}
\|(w, z)\|^{2}= & \sum_{i=2}^{3}\left[\lambda k_{i}\left|w_{i}\left(\ell_{i}\right)\right|^{2}-\lambda k_{i} \overline{w_{i}\left(\ell_{i}\right)} u_{i}\left(\ell_{i}\right)-k_{i} \overline{w_{i}\left(\ell_{i}\right)} f_{i}\left(\ell_{i}\right)\right] \\
& +\left[h \lambda\left|w_{1}(0)\right|^{2}+\overline{w_{1}(0)} u_{1}(0)-h \overline{w_{1}(0)} f_{1}(0)\right]
\end{aligned}
$$

so we have

$$
\begin{aligned}
\|(w, z)\|^{2} \leq & \frac{1}{2} \sum_{i=2}^{3}\left[2|\lambda| k_{i}\left|w_{i}\left(\ell_{i}\right)\right|^{2}+|\lambda| k_{i}\left(\left|u_{i}\left(\ell_{i}\right)\right|^{2}+\left|w_{i}\left(\ell_{i}\right)\right|^{2}\right)+k_{i}\left(\left|f_{i}\left(\ell_{i}\right)\right|^{2}+\left|w_{i}\left(\ell_{i}\right)\right|^{2}\right)\right] \\
& +\frac{1}{2}\left[(2 h|\lambda|+h+1)\left|w_{1}(0)\right|^{2}+\left|u_{1}(0)\right|^{2}+h\left|f_{1}(0)\right|^{2}\right] \\
\leq & \frac{K(|\lambda|+1)}{2} \sum_{i=2}^{3}\left|w_{i}\left(\ell_{i}\right)\right|^{2}+\frac{K|\lambda|}{2} \sum_{i=2}^{3}\left|u_{i}\left(\ell_{i}\right)\right|^{2}+\frac{K}{2} \sum_{i=2}^{3}\left|f_{i}\left(\ell_{i}\right)\right|^{2}
\end{aligned}
$$

(Remark: $\left.K=\max _{i=2,3}\left\{k_{i}\right\}\right)$ 


$$
\begin{aligned}
& +\frac{1}{2}\left[(2 h|\lambda|+h+1)\left|w_{1}(0)\right|^{2}+\left|u_{1}(0)\right|^{2}+h\left|f_{1}(0)\right|^{2}\right] \\
\leq & \frac{K(|\lambda|+1)}{2} \sum_{i=2}^{3}\left|w_{i}\left(\ell_{i}\right)\right|^{2}+\frac{(2 h|\lambda|+h+1)}{2}\left|w_{1}(0)\right|^{2} \\
& +\left(\frac{(|\lambda|+1) C}{|\lambda|}+C\right)\|(f, g)\|^{2},
\end{aligned}
$$

where $C$ is a positive constant. Obviously, if we can prove $|\lambda|\left|w_{1}(0)\right|^{2}$ and $|\lambda|\left|w_{i}\left(\ell_{i}\right)\right|^{2}, i=$ 2, 3 are bounded, the first step is then completed. satisfies

By the definition of $(w, z)$ in (4.1), we have that $z_{i}=-\lambda w_{i}(i=1,2,3)$ and $w_{i}$

$$
\begin{aligned}
& -\lambda^{2} \rho_{i}(x) w_{i}(x)+\left[\left(\sigma_{i}(x) w_{i}^{\prime}(x)\right)^{\prime}-q_{i}(x) w_{i}(x)\right]=0, \quad x \in\left(0, \ell_{i}\right), i=1,2,3 \\
& w_{1}\left(\ell_{1}\right)=0 \\
& \sigma_{i}\left(\ell_{i}\right) w_{i}^{\prime}\left(\ell_{i}\right)=-\lambda k_{i}\left(w_{i}\left(\ell_{i}\right)+u_{i}\left(\ell_{i}\right)\right)+k_{i} f_{i}\left(\ell_{i}\right), i=2,3 \\
& w_{1}(0)=w_{2}(0)=w_{3}(0) \\
& \sum_{i=1}^{3} \sigma_{i}(0) w_{i}^{\prime}(0)=\lambda h\left(w_{1}(0)+u_{1}(0)\right)-h f_{1}(0) .
\end{aligned}
$$

To estimate $\left|w_{1}(0)\right|^{2}$ and $|\lambda|\left|w_{i}\left(\ell_{i}\right)\right|^{2}, i=2,3$, we convert equation (4.3) into a standard form by introducing a new variable $\xi_{i}(x)$ and two new functions $y_{i}(x), s_{i}\left(\xi_{i}\right)$, $i=1,2,3$,

$$
\begin{aligned}
& \xi_{i}(x)=\int_{0}^{x} \sqrt{\frac{\rho_{i}(t)}{\sigma_{i}(t)}} d t, y_{i}(x)=\left(\sigma_{i}(x)\right)^{1 / 2} w_{i}(x), \\
& s_{i}\left(\xi_{i}\right)=\frac{1}{\sqrt{x_{\xi_{i}}}} y_{i}\left(x\left(\xi_{i}\right)\right), \text { for } x \in\left(0, \ell_{i}\right), i=1,2,3 .
\end{aligned}
$$

With the help of these functions, (4.3) can be rewritten into the standard form:

$$
s_{i}^{\prime \prime}\left(\xi_{i}\right)-\lambda^{2} s_{i}\left(\xi_{i}\right)=\left[b_{i}\left(\xi_{i}\right)-\phi_{i}\left(\xi_{i}\right)\right] s_{i}\left(\xi_{i}\right), \quad \xi_{i} \in\left(0, m_{i}\right), i=1,2,3,
$$

where functions $s_{i}(\cdot), i=1,2,3$ with variable $\xi_{i}$ satisfy conditions

$$
\begin{aligned}
& s_{1}\left(m_{1}\right)=0 \\
& s_{i}^{\prime}\left(m_{i}\right)+\left[c_{i}\left(\ell_{i}\right)+\lambda k_{i}\left(\frac{\rho_{i}\left(\ell_{i}\right)}{\sigma_{i}\left(\ell_{i}\right)}\right)^{-1 / 2}\right] s_{i}\left(m_{i}\right) \\
& \quad=k_{i}\left(\frac{\sigma_{i}\left(\ell_{i}\right)^{3 / 4}}{\rho_{i}\left(\ell_{i}\right)^{1 / 4}}\right)\left(-\lambda u_{i}\left(\ell_{i}\right)+f_{i}\left(\ell_{i}\right)\right), i=2,3 \\
& \left(\rho_{1}(0) \sigma_{1}(0)\right)^{-1 / 4} s_{1}(0)=\left(\rho_{2}(0) \sigma_{2}(0)\right)^{-1 / 4} s_{2}(0)=\left(\rho_{3}(0) \sigma_{3}(0)\right)^{-1 / 4} s_{3}(0) \\
& \sum_{i=1}^{3}\left(\rho_{i}(0) \sigma_{i}(0)\right)^{1 / 4} s_{i}^{\prime}(0)+\sum_{i=1}^{3} d_{i}(0) s_{i}(0)-\lambda h\left(\rho_{1}(0) \sigma_{1}(0)\right)^{-1 / 4} s_{1}(0) \\
& =h\left[\lambda u_{1}(0)-f_{1}(0)\right]
\end{aligned}
$$


where $b_{i}\left(x\left(\xi_{i}\right)\right), m_{i}, \phi_{i}\left(\xi_{i}\right), c_{i}$ and $d_{i}$ are given by (3.7), (3.12), (3.13), (3.19) and (3.20), respectively.

Equation (4.8) has two linearly independent solutions, $F\left(\lambda, \xi_{i}\right)$ and $\Psi\left(\lambda, \xi_{i}\right)$ for each index $i, i=1,2,3$. Both have asymptotic representations given by (3.21) and (3.22) according to ([11], p. 49). Consequently, the general solution $s_{i}\left(\lambda, \xi_{i}\right)$ of equation (4.8) has an asymptotic formula

$$
\begin{aligned}
s_{i}\left(\lambda, \xi_{i}\right) & =A_{i}(\lambda) F\left(\lambda, \xi_{i}\right)+B_{i}(\lambda) \Psi\left(\lambda, \xi_{i}\right) \\
& :=A_{i}(\lambda) e^{\lambda \xi_{i}}[1]_{1}+B_{i}(\lambda) e^{-\lambda \xi_{i}}[1]_{1}, \quad \xi_{i} \in\left(0, m_{i}\right), \quad i=1,2,3,
\end{aligned}
$$

where $[1]_{1}=1+O\left(\lambda^{-1}\right)$.

Now, we calculate asymptotic values of $s_{2}(0)$ and $s_{i}\left(m_{i}\right), i=2,3$. Substitute (4.13) into (4.9)-(4.12) to yield

$$
\Delta(\lambda) D(\lambda)=\beta
$$

where $\Delta(\lambda)$ is given by (3.29), $D(\lambda)=\left(A_{1}(\lambda), A_{2}(\lambda), A_{3}(\lambda), B_{1}(\lambda), B_{2}(\lambda), B_{3}(\lambda)\right)^{T}$ and vector $\beta=\left(\beta_{1}, \cdots, \beta_{6}\right)^{T}$ with entries

$$
\begin{aligned}
& \beta_{1}=\beta_{4}=\beta_{5}=0 ; \\
& \beta_{i}=k_{i}\left(\frac{\sigma_{i}\left(\ell_{i}\right)^{3 / 4}}{\rho_{i}\left(\ell_{i}\right)^{1 / 4}}\right)\left(-\lambda u_{i}\left(\ell_{i}\right)+f_{i}\left(\ell_{i}\right)\right), \quad i=2,3 ; \\
& \beta_{6}=h\left[\lambda u_{1}(0)-f_{1}(0)\right],
\end{aligned}
$$

which implies

$$
A_{i}(\lambda)=\frac{\operatorname{det} D_{i}(\lambda)}{\operatorname{det} \Delta(\lambda)}, \quad B_{i}(\lambda)=\frac{\operatorname{det} D_{i+3}(\lambda)}{\operatorname{det} \Delta(\lambda)}, i=1,2,3,
$$

where $D_{i}(\lambda)$ denotes the matrix replaced the $i_{t h}$ column in $\Delta(\lambda)$ by $\beta$.

Recall the notations $H_{i}^{ \pm}, K_{i}^{ \pm}$and $\widehat{\rho}_{i}$ defined by (3.28) and denote $P=$ $K_{2}^{-} K_{3}^{-} \sum_{i=1}^{3} \prod_{j=1, j \neq i}^{3} \widehat{\rho}_{j} H_{i}^{-}$. We calculate $A_{i}(\lambda), B_{i}(\lambda)$ directly to obtain

$$
\begin{aligned}
& A_{2}(\lambda)=-\frac{\widehat{\rho}_{1} \widehat{\rho}_{3}}{P} h u_{1}(0)+o(1), \quad A_{3}(\lambda)=-\frac{\widehat{\rho}_{1} \widehat{\rho}_{2}}{P} h u_{1}(0)+o(1), \\
& B_{2}(\lambda)=\frac{\sigma_{2}\left(\ell_{2}\right)^{3 / 4}}{\rho_{2}\left(\ell_{2}\right)^{1 / 4} K_{2}^{-}} k_{2} u_{2}\left(\ell_{2}\right) e^{\lambda m_{2}}+o\left(e^{\lambda m_{2}}\right), \\
& B_{3}(\lambda)=\frac{\sigma_{3}\left(\ell_{3}\right)^{3 / 4}}{\rho_{3}\left(\ell_{3}\right)^{1 / 4} K_{3}^{-}} k_{3} u_{3}\left(\ell_{3}\right) e^{\lambda m_{3}}+o\left(e^{\lambda m_{3}}\right),
\end{aligned}
$$

where $o(1)$ means that function $f(\lambda)$ is a higher term when $\lambda \rightarrow-\infty$.

Substituting the above formula into (4.13), we get

$$
\begin{aligned}
s_{2}(0) & =\left[-\frac{\widehat{\rho}_{1} \widehat{\rho}_{3}}{P} h u_{1}(0)+\frac{\sigma_{2}\left(\ell_{2}\right)^{3 / 4}}{\rho_{2}\left(\ell_{2}\right)^{1 / 4} K_{2}^{-}} k_{2} u_{2}\left(\ell_{2}\right)+o(1)\right]\left[1+O\left(\frac{1}{\lambda}\right)\right], \\
s_{2}\left(m_{2}\right) & =-\frac{\widehat{\rho}_{1} \widehat{\rho}_{3}}{P} h e^{\lambda m_{2}}\left[1+O\left(\frac{1}{\lambda}\right)\right] u_{1}(0)+\frac{\sigma_{2}\left(\ell_{2}\right)^{3 / 4} k_{2}}{\rho_{2}\left(\ell_{2}\right)^{1 / 4} K_{2}^{-}}\left[1+O\left(\frac{1}{\lambda}\right)\right] u_{2}\left(\ell_{2}\right)+o(1) .
\end{aligned}
$$


Therefore, we obtain estimates

$$
\begin{aligned}
\left|s_{2}(0)\right|^{2} & \leq 2\left[\frac{\widehat{\rho}_{1}^{2} \widehat{\rho}_{3}^{2} h^{2}}{P^{2}}\left|u_{1}(0)\right|^{2}+\frac{\sigma_{2}\left(\ell_{2}\right)^{3 / 2} k_{2}^{2}}{\rho_{2}\left(\ell_{2}\right)^{1 / 2}\left(K_{2}^{-}\right)^{2}}\left|u_{2}\left(\ell_{2}\right)\right|^{2}\right]\left[1+O\left(\frac{1}{\lambda}\right)\right]+o(1) \\
& \left.\leq \frac{2}{\lambda^{2}}\left[\frac{\widehat{\rho}_{1}^{2} \widehat{\rho}_{3}^{2} h^{2}}{P^{2}}+\frac{\sigma_{2}\left(\ell_{2}\right)^{3 / 2} k_{2}^{2}}{\rho_{2}\left(\ell_{2}\right)^{1 / 2}\left(K_{2}^{-}\right)^{2}}\right] \| f, g\right) \|^{2}+o(1)
\end{aligned}
$$

and

$$
\begin{aligned}
\left|s_{2}\left(m_{2}\right)\right|^{2} & \leq 2\left(\frac{\widehat{\rho}_{1}^{2} \widehat{\rho}_{3}^{2} h^{2}}{P^{2}} e^{2 \lambda m_{2}}\left|u_{1}(0)\right|^{2}+\frac{\sigma_{2}\left(\ell_{2}\right)^{3 / 2} k_{2}^{2}}{\rho_{2}\left(\ell_{2}\right)^{1 / 2}\left(K_{2}^{-}\right)^{2}}\left|u_{2}\left(\ell_{2}\right)\right|^{2}\right)\left[1+O\left(\frac{1}{\lambda}\right)\right]+o(1) \\
& \leq \frac{2}{\lambda^{2}}\left(\frac{\widehat{\rho}_{1}^{2} \widehat{\rho}_{3}^{2} h^{2}}{P^{2}} e^{2 \lambda m_{2}}+\frac{\sigma_{2}\left(\ell_{2}\right)^{3 / 2} k_{2}^{2}}{\rho_{2}\left(\ell_{2}\right)^{1 / 2}\left(K_{2}^{-}\right)^{2}}\right)\|(f, g)\|^{2}\left[1+O\left(\frac{1}{\lambda}\right)\right]+o(1) .
\end{aligned}
$$

Similarly, we have

$$
\left|s_{3}\left(m_{3}\right)\right|^{2} \leq \frac{2}{\lambda^{2}}\left(\frac{\widehat{\rho}_{1}^{2} \widehat{\rho}_{2}^{2} h^{2}}{P^{2}} e^{2 \lambda m_{3}}+\frac{\sigma_{3}\left(\ell_{3}\right)^{3 / 2} k_{3}^{2}}{\rho_{3}\left(\ell_{3}\right)^{1 / 2}\left(K_{3}^{-}\right)^{2}}\right)\|(f, g)\|^{2}\left[1+O\left(\frac{1}{\lambda}\right)\right]+o(1) .
$$

Note that

$$
w_{i}\left(x\left(\xi_{i}\right)\right)=\left(\rho_{i}\left(x\left(\xi_{i}\right)\right) \sigma_{i}\left(x\left(\xi_{i}\right)\right)\right)^{-1 / 4} s_{i}\left(\xi_{i}\right), \quad e^{2 \lambda m_{i}}=o\left(\lambda^{-1}\right), \quad i=2,3, \lambda \rightarrow-\infty .
$$

Thus, we have estimates

$$
\begin{aligned}
|\lambda|\left|w_{i}\left(\ell_{i}\right)\right|^{2} & =\left(\rho_{i}\left(\ell_{i}\right) \sigma_{i}\left(\ell_{i}\right)\right)^{-1 / 2}|\lambda|\left|s_{i}\left(m_{i}\right)\right|^{2} \leq|\lambda|^{-1}\|(f, g)\|^{2}+O(1) \\
& \leq O(1)\|(f, g)\|^{2}, \quad i=2,3 \\
|\lambda|\left|w_{1}(0)\right|^{2} & =\left|\lambda \| w_{2}(0)\right|^{2}=\left(\rho_{2}(0) \sigma_{2}(0)\right)^{-1 / 2}|\lambda|\left|s_{2}(0)\right|^{2} \\
& \leq O(1)\|(f, g)\|^{2} .
\end{aligned}
$$

Returning to (4.2), we get $\|(w, z)\|^{2} \leq O(1)\|(f, g)\|^{2}$. Therefore, there is a constant $M>0$ such that

$$
\left\|R\left(\lambda, \mathcal{A}^{*}\right)(f, g)\right\| \leq\|(w, z)\|+\left\|R\left(\lambda, \mathcal{A}_{0}^{*}\right)(f, g)\right\| \leq M\|(f, g)\|
$$

when $\lambda \in \rho\left(\mathcal{A}^{*}\right) \cap \mathbb{R}_{-}$.

Step 2.

$$
R\left(\lambda, \mathcal{A}^{*}\right)(f, g)=\frac{H(\lambda ; f, g)}{M(\lambda)}
$$

where $H(\lambda ; f, g)$ is an $\mathcal{H}$-value entire function of finite exponential type and $M(\lambda)$ is a scalar entire function of finite exponential type. 
In fact, given $\lambda \in \rho(\mathcal{A}), \lambda \neq 0,\left(\lambda I-\mathcal{A}^{*}\right)^{-1}(f, g)=:(\tilde{u}, \tilde{v}) \in D\left(\mathcal{A}^{*}\right)$ for any $(f, g) \in$ $\mathcal{H}$, which implies that $\tilde{v}_{i}(x)=f_{i}(x)-\lambda \tilde{u}_{i}(x), i=1,2,3$ with $\tilde{u}_{i}(x)$ satisfying

$$
\begin{aligned}
& \left(\sigma_{i}(x) \tilde{u}_{i}^{\prime}(x)\right)^{\prime}-\left[\lambda^{2} \rho_{i}(x)+q_{i}(x)\right] \tilde{u}_{i}(x)=\rho_{i}(x)\left[g_{i}(x)-\lambda f_{i}(x)\right], x \in\left(0, \ell_{i}\right) \\
& \tilde{u}_{1}\left(\ell_{1}\right)=0 \\
& \tilde{u}_{1}(0)=\tilde{u}_{2}(0)=\tilde{u}_{3}(0) \\
& \sigma_{i}\left(\ell_{i}\right) \tilde{u}_{i}^{\prime}\left(\ell_{i}\right)=-k_{i} \lambda \tilde{u}_{i}\left(\ell_{i}\right)+k_{i} f_{i}\left(\ell_{i}\right), i=2,3 \\
& \sum_{i=1}^{3} \sigma_{i}(0) \tilde{u}_{i}^{\prime}(0)=-h\left[f_{1}(0)-\lambda \tilde{u}(0)\right]
\end{aligned}
$$

The theory of solution of the ordinary differential equations asserts that function $\tilde{u}_{i}(x, \lambda)$ is the meromorphic function of finite exponential type in $\lambda$. Therefore, $R\left(\lambda, \mathcal{A}^{*}\right)(f, g)=\left(\widetilde{u}_{i}, f_{i}-\lambda \widetilde{u}_{i}\right)_{i=1,2,3}$ also is an $\mathcal{H}$-valued meromorphic function in $\lambda$.

Step 3. The system of generalised eigenvectors of $\mathcal{A}$ is complete in $\mathcal{H}$.

Since $\mathcal{A}$ generates a $C_{0}$-semigroup of contraction on $\mathcal{H}$, the same applies to $\mathcal{A}^{*}$. It always holds that $\left\|R\left(\lambda, \mathcal{A}^{*}\right)\right\| \leq \frac{1}{\Re \lambda \lambda}, \Re \lambda>0$, which implies that $R\left(\lambda, \mathcal{A}^{*}\right) \rightarrow 0$ as $\Re \lambda \rightarrow$ $\infty$. Note that the order of the entire function of finite exponential type is 1 . We take $\rho_{2}=\rho_{1}=\rho=1$, the rays $\gamma_{0}=-N+i y, \gamma_{1}=-N-y, \gamma_{2}=-N-i y$ for $y \in(0, \infty)$ with $N$ being large enough. The results of Steps 1 and 2 show that all conditions in Proposition 4.1 are fulfilled. The desired result follows from Proposition 4.1.

Now, we are in a position to discuss the basis generation of the eigenvectors and generalised eigenvectors of $\mathcal{A}$. Let us recall the notion of Riesz basis of subspaces [16].

Let $\mathcal{H}$ be a Hilbert space and $\left\{H_{k}\right\}_{k=1}^{\infty}$ be a sequence of subspaces of $\mathcal{H}$. The sequence $\left\{H_{k}\right\}_{k=1}^{\infty}$ is said to be a Riesz basis of subspaces if any $x \in \mathcal{H}$ can be uniquely represented as a series $x=\sum_{k=1}^{\infty} x_{k}, x_{k} \in H_{k}$ and there exist positive constants $C_{1}$ and $C_{2}$ such that

$$
C_{1} \sum_{k=1}^{\infty}\left\|x_{k}\right\|^{2} \leq\|x\|^{2} \leq C_{2} \sum_{k=1}^{\infty}\left\|x_{k}\right\|^{2}, \quad \forall x \in \mathcal{H}
$$

A sequence $\left\{f_{i}\right\}_{i=1}^{\infty} \subset \mathcal{H}$ is called a Riesz basis with parentheses for a Hilbert space $\mathcal{H}$, if there is a sequence of integers $n_{0}=1 \leq n_{1}<\cdots<n_{k}<\cdots$ such that the following conditions hold:

(1) $\left\{f_{i}\right\}_{i=1}^{\infty}$ is complete in $\mathcal{H}$;

(2) the subspaces $H_{k}=\operatorname{span}\left\{f_{i}, n_{k-1} \leq i \leq n_{k}-1\right\}$ is a Riesz basis of subspaces of $\mathcal{H}($ see $[13])$.

The following proposition gives a sufficient condition for Riesz basis with parentheses, which is from [20] and is an extension result of [21].

Proposition 4.2. Let $\mathcal{A}$ be the generator of a $C_{0}$-semigroup $T(t)(t \geq 0)$ on a separable Hilbert space $\mathcal{H}$. Suppose that the following conditions are satisfied:

(1) The spectrum of $\mathcal{A}$ has a decomposition $\sigma(\mathcal{A})=\sigma_{1}(\mathcal{A}) \cup \sigma_{2}(\mathcal{A})$;

(2) There is a real number $\alpha \in \mathbb{R}$ such that $\sup \left\{\Re \lambda \mid \lambda \in \sigma_{1}(\mathcal{A})\right\} \leq \alpha \leq \inf \{\Re \lambda \mid \lambda \in$ $\left.\sigma_{2}(\mathcal{A})\right\}$

(3) $\sigma_{2}(\mathcal{A})=\left\{\lambda_{k}\right\}_{k \in \mathbb{N}}$ consists of isolated eigenvalues of $\mathcal{A}$ and is a union of finitely separated sets. 
Then, there are two $T(t)$-invariant closed subspaces $\mathcal{H}_{1}$ and $\mathcal{H}_{2}$

$$
\mathcal{H}_{1}=\left\{f \in \mathcal{H} \mid E(\lambda, \mathcal{A}) f=0, \forall \lambda \in \sigma_{2}(\mathcal{A})\right\}, \mathcal{H}_{2}=\overline{\operatorname{span}\left\{E\left(\lambda_{k}, \mathcal{A}\right) f: \lambda_{k} \in \sigma(\mathcal{A}), \forall f \in \mathcal{H}\right\}}
$$

and $\mathcal{H}_{1} \bigcap \mathcal{H}_{2}=\{0\}$ with property that $\sigma\left(\left.\mathcal{A}\right|_{\mathcal{H}_{1}}\right)=\sigma_{1}(\mathcal{A})$ and $\sigma\left(\left.\mathcal{A}\right|_{\mathcal{H}_{2}}\right)=\sigma_{2}(\mathcal{A})$. Moreover, there is a finite collection $\Omega_{k}$ of elements in $\sigma_{2}(\mathcal{A})$ such that $\left\{E\left(\Omega_{k}, \mathcal{A}\right) H_{2}\right\}_{k \in \mathbb{N}}$ forms a subspace Riesz basis for $\mathcal{H}_{2}$, where $E\left(\Omega_{k}, \mathcal{A}\right)=\sum_{\lambda \in \Omega_{k}} E(\lambda, \mathcal{A})$ is the Riesz projector corresponding to $\Omega_{k}$.

THEOREM 4.2. Let $\mathcal{A}$ be defined by (2.1)-(2.2). Suppose that $k_{i}(i=2,3)$ and $h$ satisfy (3.31). Then, there is a sequence of generalised eigenvectors of $\mathcal{A}$ that forms a Riesz basis with parentheses for $\mathcal{H}$.

Proof. We take $\sigma_{1}(\mathcal{A})=\{-\infty\}, \sigma_{2}(\mathcal{A})=\sigma_{p}(\mathcal{A})$, then $\sigma(\mathcal{A})=\sigma_{1}(\mathcal{A}) \cup \sigma_{2}(\mathcal{A})$. Condition (1) in Proposition 4.2 is satisfied. Theorem 3.1 ensures that the spectrum of $\mathcal{A}$ is distributed in a strip parallel to the imaginary axis and $\sigma(\mathcal{A})$ is an union of finitely many separable sets, which together with Corollary 2.1 imply the conditions (2) and (3) of Proposition 4.2. Hence, there is a sequence of generalised eigenvectors of $\mathcal{A}$ that forms a subspace Riesz basis (i.e. Riesz basis with parentheses we defined above) for $\mathcal{H}_{2}$ according to Proposition 4.2. Furthermore, the completeness of generalised eigenvectors of $\mathcal{A}$ in Theorem 4.1 implies that $\mathcal{H}_{2}=\mathcal{H}$. The proof is then complete.

We note that the Riesz basis with parentheses is not a basis for $\mathcal{H}$ in the sense of Schauder basis. In general, the property of Riesz basis with parentheses cannot ensure the spectrum-determined growth assumption. However, under certain conditions of spectral distribution, the Riesz basis with parentheses can assert that the spectrumdetermined growth condition holds. The spectral condition is just as $\sigma_{2}(\mathcal{A})$ stated in Proposition 4.2; its proof can refer to [21]. Based on this fact, we can assert the stability by the spectral distribution.

THEOREM 4.3. Let damping constants $k_{i}, i=2,3$ and $h \in \mathbb{R}$ satisfy (3.31). Then, the system (2.3) is exponentially stable for $k_{i}>0$ and $h \geq 0$.

Proof. As a direct result of Theorem 4.2 and Theorem 3.1, we have $\omega(\mathcal{A})=\sup \{\Re \lambda \mid \lambda \in \sigma(\mathcal{A})\}$. Obviously, if $\sup \{\Re \lambda \mid \lambda \in \sigma(\mathcal{A})\}<0$, then system $(2.3)$ is exponentially stable.

In what follows, we prove that

$$
\sup \{\Re \lambda \mid \lambda \in \sigma(\mathcal{A})\}<0 .
$$

First, $\mathcal{A}$ is the generator of a $C_{0}$-semigroup of contractions on $\mathcal{H}$ and $\sigma(\mathcal{A})=$ $\sigma_{p}(\mathcal{A})=\{\lambda \in \mathbb{C} \mid \operatorname{det} \Delta(\lambda)=0\}$ (see, Theorem 3.1). We have $\sup \{\Re \lambda \mid \lambda \in \sigma(\mathcal{A})\} \leq 0$.

Next, there is no eigenvalue on the imaginary axis. In fact, if there is $(u, v) \in$ $\mathcal{D}(\mathcal{A})$ such that $\mathcal{A}(u, v)=\lambda(u, v), \lambda \in i \mathbb{R}$, then from the dissipation of $\mathcal{A}$, we get $\Re \lambda\|(u, v)\|^{2}=-\sum_{i=2}^{3} k_{i}\left|u_{i}\left(\ell_{i}\right)\right|^{2}-h\left|v_{1}(0)\right|^{2}=0$, which implies $u\left(\ell_{i}\right)=0, i=2,3$ and $v_{1}(0)=0$. From $\mathcal{A}(u, v)=\lambda(u, v)$, we deduce that $v=\lambda u$ and $u=\left\{u_{i}\right\}$ satisfies the 
equations

$$
\begin{aligned}
& \lambda^{2} \rho_{i}(x) u_{i}(x)-\left[\left(\sigma_{i}(x) u_{i}^{\prime}(x)\right)^{\prime}-q_{i}(x) u_{i}(x)\right]=0, x \in\left(0, \ell_{i}\right), i=1,2,3 \\
& u_{i}\left(\ell_{i}\right)=u_{i}(0)=0, \quad i=1.2,3 \\
& \sigma_{i}\left(\ell_{i}\right) u_{i}^{\prime}\left(\ell_{i}\right)=-k_{i} \lambda u_{i}\left(\ell_{i}\right)=0, \quad i=2,3 \\
& \sum_{i=1}^{3} \sigma_{i}(0) u_{i}^{\prime}(0)=\lambda h u_{1}(0)=0
\end{aligned}
$$

The uniqueness theorem for a solution of an ordinary differential equation asserts that the above equations have uniquely zero solutions, so $(u, v)=0$. Therefore, there is no eigenvalue on the imaginary axis.

Finally, the imaginary axis is not an asymptote of $\sigma(\mathcal{A})$. By contradictory, if there is a sequence $\left\{\lambda_{n}\right\} \subset \sigma(\mathcal{A})$ with $\Re \lambda_{n} \rightarrow 0$ and $\left|\lambda_{n}\right| \rightarrow \infty$ such that $\operatorname{det} \Delta\left(\lambda_{n}\right)=0, \forall n \in \mathbb{N}$, let $\lambda_{n}=\alpha_{n}+i \beta_{n}$, then we have

$$
\operatorname{det} \Delta\left(\lambda_{n}\right)-\operatorname{det} \Delta\left(i \beta_{n}\right)=\left[\operatorname{det} \Delta\left(\theta_{n}+i \beta_{n}\right)\right]^{\prime} \alpha_{n}, \quad \theta_{n} \in\left(\alpha_{n}, 0\right)
$$

The property of det $\Delta(\lambda)$ of sine-type ensures [det $\left.\Delta\left(\theta_{n}+i \beta_{n}\right)\right]^{\prime}$ is uniformly bounded, so it holds that $\lim _{n \rightarrow \infty} \operatorname{det} \Delta\left(\lambda_{n}\right)=\lim _{n \rightarrow \infty} \operatorname{det} \Delta\left(i \beta_{n}\right)=0$. If the main part of $\Delta(\lambda)$ is denoted by $\Delta_{0}(\lambda)$, i.e.,

$$
\Delta_{0}(\lambda)=\left(\begin{array}{cccccc}
e^{\lambda m_{1}} & 0 & 0 & e^{-\lambda m_{1}} & 0 & 0 \\
0 & K_{2}^{+} e^{\lambda m_{2}} & 0 & 0 & K_{2}^{-} e^{-\lambda m_{2}} & 0 \\
0 & 0 & K_{3}^{+} e^{\lambda m_{3}} & 0 & 0 & K_{3}^{-} e^{-\lambda m_{3}} \\
\widehat{\rho}_{1} & -\widehat{\rho}_{2} & 0 & \widehat{\rho}_{1} & -\widehat{\rho}_{2} & 0 \\
\widehat{\rho}_{1} & 0 & -\widehat{\rho}_{3} & \widehat{\rho}_{1} & 0 & -\widehat{\rho}_{3} \\
H_{1}^{-} & H_{2}^{-} & H_{3}^{-} & -H_{1}^{+} & -H_{2}^{+} & -H_{3}^{+}
\end{array}\right)
$$

then we have

$$
\lim _{n \rightarrow \infty} \operatorname{det} \Delta\left(i \beta_{n}\right)=\lim _{n \rightarrow \infty} \operatorname{det} \Delta_{0}\left(i \beta_{n}\right)=0
$$

which implies that

$$
\inf _{x \in \mathbb{R}}\left|\operatorname{det} \Delta_{0}(i x)\right|=0
$$

In what follows, we prove that inf $\operatorname{se\mathbb {R}}_{x}\left|\operatorname{det} \Delta_{0}(i x)\right| \neq 0$. For simplicity of notation, we denote $\widehat{k}_{i}=k_{i}\left(\frac{\rho_{i}\left(\ell_{i}\right)}{\sigma_{i}\left(\ell_{i}\right)}\right)^{-1 / 2} \frac{1}{\sigma_{i}\left(\ell_{i}\right)}$ and $\mu_{i}=\sqrt{\rho_{i}(0) \sigma_{i}(0)}$. Then, the coefficients in (4.23) can be rewritten into follows:

$$
\begin{aligned}
& K_{i}^{+}=\sigma_{i}\left(\ell_{i}\right)\left(\widehat{k_{i}}+1\right), \quad K_{i}^{-}=\sigma_{i}\left(\ell_{i}\right)\left(\widehat{k_{i}}-1\right), i=2,3, \\
& H_{1}^{-}=\widehat{\rho}_{1}\left(\mu_{1}-h\right), \quad H_{1}^{+}=\widehat{\rho}_{1}\left(\mu_{1}+h\right), \\
& H_{i}^{ \pm}=\sqrt{\mu_{i}}, \quad i=2,3, \widehat{\rho}_{i}=\left(\sqrt{\mu_{i}}\right)^{-1}, i=1,2,3 .
\end{aligned}
$$


A direct calculation gives

$$
\begin{aligned}
& \frac{\operatorname{det} \Delta_{0}(\lambda)}{\widehat{\sigma_{2}\left(\ell_{2}\right) \sigma_{3}\left(\ell_{3}\right) \widehat{\rho}_{1} \widehat{\rho}_{2} \widehat{\rho}_{3}}} \\
= & -2 e^{\lambda\left(m_{2}+m_{3}\right)}\left(\widehat{k_{2}}+1\right)\left(\widehat{k_{3}}+1\right)\left\{\mu_{1} \cosh \lambda m_{1}+\left[h+\mu_{2}+\mu_{3}\right] \sinh \lambda m_{1}\right\} \\
& -2 e^{-\lambda\left(m_{2}+m_{3}\right)}\left(\widehat{k_{2}}-1\right)\left(\widehat{k_{3}}-1\right)\left\{\mu_{1} \cosh \lambda m_{1}+\left[h-\mu_{2}-\mu_{3}\right] \sinh \lambda m_{1}\right\} \\
& +2 e^{\lambda\left(m_{2}-m_{3}\right)}\left(\widehat{k_{2}}+1\right)\left(\widehat{k_{3}}-1\right)\left\{\mu_{1} \cosh \lambda m_{1}+\left[h+\mu_{2}-\mu_{3}\right] \sinh \lambda m_{1}\right\} \\
& +2 e^{-\lambda\left(m_{2}-m_{3}\right)}\left(\widehat{k_{2}}-1\right)\left(\widehat{k_{3}}+1\right)\left\{\mu_{1} \cosh \lambda m_{1}+\left[h-\mu_{2}+\mu_{3}\right] \sinh \lambda m_{1}\right\} .
\end{aligned}
$$

Therefore, for any $x \in \mathbb{R}$, we have

$$
\begin{aligned}
\frac{\operatorname{det} \Delta_{0}(i x)}{\sigma_{2}\left(\ell_{2}\right) \sigma_{3}\left(\ell_{3}\right) \widehat{\rho}_{1} \widehat{\rho}_{2} \widehat{\rho}_{3}}= & -2 \mu_{1} \cos x m_{1} \cdot\left(\cos x m_{2}+i \hat{k}_{2} \sin x m_{2}\right)\left(\cos x m_{3}+i \hat{k_{3}} \sin x m_{3}\right) \\
& -2 i \sin x m_{1} \cdot\left\{h\left(\cos x m_{2}+i \hat{k}_{2} \sin x m_{2}\right)\left(\cos x m_{3}+i \hat{k_{3}} \sin x m_{3}\right)\right. \\
& +\mu_{2}\left(\hat{k}_{2} \cos x m_{2}+i \sin x m_{2}\right)\left(\cos x m_{3}+i \hat{k_{3}} \sin x m_{3}\right) \\
& \left.+\mu_{3}\left(\cos x m_{2}+i \hat{k}_{2} \sin x m_{2}\right)\left(\hat{k}_{3} \cos x m_{3}+i \sin x m_{3}\right)\right\} .
\end{aligned}
$$

Set

$$
M(x)=\cos x m_{2}+i \hat{k}_{2} \sin x m_{2}, \quad N(x)=\cos x m_{3}+i \hat{k}_{3} \sin x m_{3} .
$$

Obviously,

$$
0<\min \left\{1, \hat{k}_{2}\right\} \leq|M(x)| \leq\left(1+\hat{k}_{2}\right), \quad 0<\min \left\{1, \hat{k}_{3}\right\} \leq|N(x)| \leq\left(1+\hat{k}_{3}\right) .
$$

Now, we calculate

$$
\begin{aligned}
\frac{\operatorname{det} \Delta_{0}(i x)}{-2} \sigma_{2}\left(\ell_{2}\right) \sigma_{3}\left(\ell_{3}\right) \widehat{\rho}_{1} \widehat{\rho}_{2} \widehat{\rho}_{3} M(x) N(x) \\
=\mu_{1} \cos x m_{1}+i \sin x m_{1} \cdot\left\{h+\frac{\mu_{2}}{|M(x)|^{2}}\left[\hat{k}_{2}+i \frac{1-\hat{k}_{2}^{2}}{2} \sin 2 x m_{2}\right]\right. \\
\left.\quad+\frac{\mu_{3}}{|N(x)|^{2}}\left[\hat{k}_{3}+i \frac{1-\hat{k}_{3}^{2}}{2} \sin 2 x m_{3}\right]\right\} \\
=\mu_{1} \cos x m_{1}+\sin x m_{1} \cdot\left(\frac{\mu_{2}}{|M(x)|^{2}} \frac{\hat{k}_{2}^{2}-1}{2} \sin 2 x m_{2}+\frac{\mu_{3}}{|N(x)|^{2}} \frac{\hat{k}_{3}^{2}-1}{2} \sin 2 x m_{3}\right) \\
\quad+i \sin x m_{1} \cdot\left(h+\hat{k}_{2} \frac{\mu_{2}}{|M(x)|^{2}}+\hat{k}_{3} \frac{\mu_{3}}{|N(x)|^{2}}\right) .
\end{aligned}
$$


Clearly,

$$
\begin{aligned}
& \left|\frac{\operatorname{det} \Delta_{0}(i x)}{-2 \sigma_{2}\left(\ell_{2}\right) \sigma_{3}\left(\ell_{3}\right) \widehat{\rho}_{1} \widehat{\rho}_{2} \widehat{\rho}_{3} M(x) N(x)}\right|^{2} \\
& \quad=\left|\mu_{1} \cos x m_{1}+\sin x m_{1} \cdot\left(\frac{\mu_{2}}{|M(x)|^{2}} \frac{\hat{k}_{2}^{2}-1}{2} \sin 2 x m_{2}+\frac{\mu_{3}}{|N(x)|^{2}} \frac{\hat{k}_{3}^{2}-1}{2} \sin 2 x m_{3}\right)\right|^{2} \\
& \quad+\left|\sin x m_{1} \cdot\left(h+\hat{k}_{2} \frac{\mu_{2}}{|M(x)|^{2}}+\hat{k}_{3} \frac{\mu_{3}}{|N(x)|^{2}}\right)\right|^{2} .
\end{aligned}
$$

From above we see that

$$
\eta=\inf _{x \in \mathbb{R}}\left|\frac{\operatorname{det} \Delta_{0}(i x)}{-2 \sigma_{2}\left(\ell_{2}\right) \sigma_{3}\left(\ell_{3}\right) \widehat{\rho}_{1} \widehat{\rho}_{2} \widehat{\rho}_{3} M(x) N(x)}\right|^{2}>0,
$$

this is because we always have inequality

$$
\left|\frac{\operatorname{det} \Delta_{0}(i x)}{-2 \sigma_{2}\left(\ell_{2}\right) \sigma_{3}\left(\ell_{3}\right) \widehat{\rho}_{1} \widehat{\rho}_{2} \widehat{\rho}_{3} M(x) N(x)}\right| \geq\left(h+\frac{\hat{k}_{2} \mu_{2}}{1+\hat{k}_{2}}+\frac{\hat{k}_{3} \mu_{3}}{1+\hat{k}_{3}}\right)\left|\sin x m_{1}\right|,
$$

if there is a sequence $x_{n} \in \mathbb{R}$ such that $\sin x_{n} m_{1} \longrightarrow 0$, then $\cos x_{n} m_{1} \longrightarrow 1$, which yields

$$
\left|\frac{\operatorname{det} \Delta_{0}\left(i x_{n}\right)}{-2 \sigma_{2}\left(\ell_{2}\right) \sigma_{3}\left(\ell_{3}\right) \rho_{1} \rho_{2} \rho_{3} M\left(x_{n}\right) N\left(x_{n}\right)}\right| \longrightarrow \mu_{1}>0
$$

Thus,

$$
\begin{aligned}
\inf _{x \in \mathbb{R}}\left|\operatorname{det} \Delta_{0}(i x)\right| & \geq 2 \sigma_{2}\left(\ell_{2}\right) \sigma_{3}\left(\ell_{3}\right) \widehat{\rho}_{1} \widehat{\rho}_{2} \widehat{\rho}_{3} \eta \cdot \inf _{x \in \mathbb{R}}|M(x)| \cdot \inf _{x \in \mathbb{R}}|N(x)| \\
& \geq 2 \sigma_{2}\left(\ell_{2}\right) \sigma_{3}\left(\ell_{3}\right) \widehat{\rho}_{1} \widehat{\rho}_{2} \widehat{\rho}_{3} \eta \cdot \min \left\{1, \hat{k}_{2}\right\} \cdot \min \left\{1, \hat{k}_{3}\right\}>0 .
\end{aligned}
$$

This contradicts (4.24). Therefore, we conclude that $\sup \{\Re \lambda \mid \lambda \in \sigma(\mathcal{A})\}<0$. The desired result follows.

ACKNOWLEDGements. The authors would like to thank the referee for his extremely helpful suggestions and comments.

\section{REFERENCES}

1. K. Ammari and M. Jellouli, Stabilization of star-shaped tree of elastic strings, Differ. Int. Equ. 17 (2004), 1395-1410.

2. K. Ammari, M. Jellouli and M. Khenissi, Stabilization of generic trees of strings, J. Dyn. Control Syst. 11(2) (2005), 177-193.

3. S. A. Avdonin and S. A. Ivanov, Families of exponentials. The method of moments in controllability problems for distributed parameter systems (Cambridge University Press, Cambridge, UK, 1995). 1976).

4. J. A. Bondy and U. S. R. Murty, Graph theory with applications (Macmillan, London,

5. G. Chen, M. C. Delfour, A. M. Krall and G. Payre, Modeling, stabilization and control of serially connected beams, SIAM J. Control Optim. 25(3) (1987), 526-546. 
6. N. Dunford and J. T. Schwartz, Linear operators, part III, spectral operators (Wileyinterscience, New York, 1971).

7. R. Dager and E. Zuazua, Wave propagation, observation and control in 1-d flexible multi-structures, Mathematiques \& Applications, vol. 50 (Springer-Verlag, Berlin, New York, 2006).

8. J. E. Lagnese, G. Leugering and E. J. P. G. Schmit, Modeling, analysis and control of dynamic elastic multi-link structures (Birkhaüser, Basel, 1994).

9. G. Leugering and E. Zuazua, Exact controllability of generic trees, in Control of systems governed by partial differential equations (Nancy, France, March 1999). ESAIM Proceeding.

10. K. S. Liu, F. L. Huang and G. Chen, Exponential stability analysis of a long chain of coupled vibrating strings with dissipative linkage, SIAM J. Appl. Math. 49 (1989), 1694-1707.

11. M. A. Naimark, Linear differential operators (Frederick Ungar, New York, 1967).

12. A. Pazy, Semigroups of linear operators and applications to partial differential equations (Springer-Verlag, New York, 1983).

13. A. S. Markus, Introduction to the spectral theory of polynormial pencils, AMS Translation of Mathematical Monographs, vol. 71 (American Mathematical Society, Providence, RI, 1988), $25-27$.

14. M. V. Keldysh, On the completeness of the eigenfunctions for certain classes of nonselfadjoint linear operatrors, Uspelhi Mta. Nauk, 27(4) (1971), (160), 15-41; English transl. in Russian Math. Serveys 27 (1971).

15. A. A. Shkalikov, Boundry problems for ordinary differential equations with parameter in the boundary conditions, J. Math. Sci. 33(6) (1986), 1311-1342.

16. I. C. Gohberg and M. G. Krein, Introduction to the theory of linear nonselfadjoint operators, AMS of Translation Mathematical Monographs, vol. 18 (American Mathematical Society, Providence, RI, 1969).

17. M. A. Shubov, Basis property of eigenfunctions of nonselfadjoint operator pencils generated by the equations of nonhomogeneous damped string, Integr. Equ. Oper. Theor. 25 (1996), 289-328.

18. J. M. Wang and B. Z. Guo, Rasis basis and stabilization for the flexible structure of a symmetric tree-shaped beam networks, Math. Meth. Appl. Sci. 31 (2008), 289-314.

19. G. Q. Xu and B. Z. Guo, Riesz basis property of evolution equations in Hilbert space and application to a coupled string equation, SIAM Control Optim. 42(3) (2003), 966-984.

20. G. Q. Xu, Z. J. Han and P. Yung, Riesz basis property of serially connected Timoshenko beams, Int. J. Control 80(3) (2007), 470-485.

21. G. Q. Xu and S. P. Yung, The expansion of semigroup and criterion of Riesz basis, J. Differ. Equ. 210 (2005), 1-24.

22. G. Q. Xu, D. Y. Liu and Y. Q. Liu, Abstract second order hyperbolic system and applications, SIAM J. Control Optim. 47(4) (2008), 1762-1784.

23. G. Q. Xu and S. P. Yung, Stability and Riesz basis property of a star-shaped network of Euler-Berboulli beams with joint damping, Netw. Heterogeneous Media. 3(4) (2008), 723-747. 